Research Paper

\title{
Correlation between ground-glass opacity on pulmonary CT and the levels of inflammatory cytokines in patients with moderate-to-severe COVID-19 pneumonia
}

\author{
Zubo $\mathrm{Wu}^{1^{*}}$, Xiaoping $\mathrm{Liu}^{2^{*}}$, Jie Liu ${ }^{1}$, Feng Zhu ${ }^{3,4}$, Yali Liu ${ }^{1}$, Yalan Liu ${ }^{\bowtie}$, Hua Peng ${ }^{1 凶}$ \\ 1. Department of Pediatrics, Union Hospital, Tongji Medical College, Huazhong University of Science and Technology, 1277 Jiefang Avenue, Wuhan, 430022 , \\ P.R. China. \\ 2. Department of Emergency and Pediatrics, Shenzhen Baoan Women's and Children's Hospital, Jinan University, Shenzhen, 518102, P.R.China \\ 3. Clinical Center of Human Gene Research, Union Hospital, Tongji Medical College, Huazhong University of Science and Technology, 1277 Jiefang Avenue, \\ Wuhan, 430022, P.R. China. \\ 4. Department of Cardiology, Tongji Medical College, Union Hospital, Huazhong University of Science and Technology, 1277 Jiefang Avenue, Wuhan, 430022 , \\ P.R. China.
}

* These authors contributed equally tot his work.

$\square$ Corresponding authors: Yalan Liu, Department of Pediatric, Union Hospital, Tongji Medical College, Huazhong University of Science and Technology, 1277 Jiefang Avenue, Wuhan, 430022, P.R. China. Email: liuyalan0923@hotmail.com. Hua Peng, Department of Pediatric, Union Hospital, Tongji Medical College, Huazhong University of Science and Technology, 1277 Jiefang Avenue, Wuhan, 430022, P.R. China. Email: PengH@whuh.com.

(c) The author(s). This is an open access article distributed under the terms of the Creative Commons Attribution License (https://creativecommons.org/licenses/by/4.0/). See http://ivyspring.com/terms for full terms and conditions.

Received: 2020.12.02; Accepted: 2021.03.31; Published: 2021.04.16

\begin{abstract}
Objectives: Comparative analysis of laboratory data in moderate-to-severe COVID-19 patients presenting with or without ground-glass opacities (GGOs).

Methods: This retrospective study examined 61 patients with moderate-to-severe COVID-19, as defined by the report of the WHO-China Joint Mission on COVID-19. All patients were admitted to the Department of Infectious Diseases, Wuhan Union Hospital from Dec 28, 2019 to Feb 22, 2020 and classified into a GGO group or a non-GGO group based on CT results. The clinical characteristics and laboratory data of the two groups were compared. Data were analyzed using univariate and multivariate analysis, and using receiver operating characteristic (ROC) analysis.

Results: Forty-five patients were in the GGO group $(73.8 \%, 21$ females, 24 males, mean age $54.8 \pm 17.8$ years) and 16 were in the non-GGO group ( $26.2 \%, 11$ females, 5 males, mean age $53 \pm 14.9$ years). The levels of IL-2, IL-4, and IFN- $y$ were greater in the GGO group (all P<0.05). ROC analysis indicated that an elevated level of IL-2 was a good predictor of GGO (area under the curve: 0.716 , optimal cutoff: 3.205 $\mathrm{pg} / \mathrm{mL}, 53.8 \%$ sensitivity, $87.5 \%$ specificity, $\mathrm{p}<0.05$ ). Multivariate analysis showed that IL-2 level was a significant and independent risk factor for lung GGO (OR: 8.167; 95\% Cl: 1.63, 40.8; $\mathrm{P}<0.05$ ).

Conclusions: There were correlations between GGO in the lungs of patients with moderate-to-severe COVID-19 and the levels of IL-2, IL-4, and INF-y. IL-2 was a significant and independent risk factor for GGO. These findings provide a basis for studying the mechanism of pulmonary lesions in COVID-19 patients.
\end{abstract}

Key words: COVID-19, SARS-CoV-2, GGO, Moderate-to-severe, Inflammatory cytokines, Risk factors.

\section{Introduction}

Researchers first reported coronavirus disease 2019 (COVID-19), which is caused by the severe acute respiratory syndrome virus 2 (SARS-CoV-2), in
Wuhan during December 2019 [1]. SARS-CoV-2 is a single-stranded positive-sense RNA virus that is in the Nidovirales order, the Coronaviridae family, and the 
Betacoronavirus genus [2]. Similar to diseases caused by related coronaviruses, SARS-CoV-1 and Middle East respiratory syndrome-related coronavirus (MERS-CoV), COVID-19 can lead to severe respiratory symptoms, but SARS-CoV-2 has much greater infectivity than these other viruses. COVID-19 is now widespread in North America and Europe.

There are currently no effective treatments for patients with COVID-19 [1, 3, 4]. The primary characteristics of COVID-19 from computed tomography (CT) imaging are bilateral pulmonary ground-glass opacity (GGO), "crazy paving" pattern, airway changes, and reversed halo sign. There may also be other manifestations, such as consolidation and interlobular septal thickening. GGO is the earliest abnormality to appear and is also the major pulmonary change [5]. A previous study reported that 73 of 81 COVID-19 patients (80\%) had GGOs [6] and another study reported that $98 \%$ of COVID-19 patients had GGOs [7]. A study of 1099 patients with acute respiratory disease (ARD) due to COVID-19 reported that $76.4 \%$ of these patients had abnormal chest $\mathrm{CT}$ image results, and that ground-glass opacity (GGO) was the most common abnormality [8].

As COVID-19 worsens, an individual GGO may increase and merge with other GGOs, and then evolve into fibrous streaks and solid nodules; alternatively, the resolution of GGOs may be related to improved patient condition $[9,10]$. A GGO on pulmonary CT manifests as a cloudy light-shadow nodule with slightly increased density and a fan-shaped distribution of pulmonary segments or lobes. An air bronchogram may be present in sheet-like shadows, or the consolidation may be accompanied by signs of bronchial ventilation [11]. A recent study of the postmortem biopsy of a COVID-19 patient identified pulmonary edema and hyaline membrane formation, and these are considered the potential pathological driving forces of the GGO [12]. However, the pathogenesis of GGO in the lungs of patients with moderate or severe COVID-19 is still unclear.

In viral pneumonia, the abnormal release of proinflammatory cytokines leads to apoptosis of lung epithelial and endothelial cells, thus destroying the pulmonary microvascular and alveolar epithelial cell barrier, leading to vascular leakage, alveolar edema, and hypoxia [13]. COVID-19 is characterized by an excessive inflammatory response and the release of a large number of pro-inflammatory cytokines. Several studies found that this cytokine storm is directly related to lung injury, multiple organ failure, and poor prognosis in patients with severe COVID-19[14, 15]. ARDS with hypoxic saturation is the main cause of death from COVID-19. Although the exact mechanism by which COVID-19 causes ARDS is not fully clear, excessive production of pro-inflammatory cytokines is considered to be one of the main factors $[10,16]$.

In this study, we examined the independent risk factors for GGO in the lungs of patients with moderate or severe COVID-19.

\section{Materials and Methods}

This study was reviewed and approved by the Institutional Review Board of Union Hospital, Huazhong University of Science \& Technology (Wuhan, China). The local Ethics Committee approved this study and each patient provided informed consent.

\section{Patients}

This single center, retrospective case-control study analyzed patients who had moderate or severe COVID-19 pneumonia and were diagnosed in our hospital between Dec 28, 2019 and Feb 22, 2020. The diagnostic criteria for moderate and severe pneumonia due to COVID-19 are based on the report of the WHO China Joint Investigation Mission on COVID-19 [17]. Moderate disease was defined as laboratory confirmed SARS-CoV-2 infection with pneumonia. Severe disease was defined by laboratory confirmed SARS-CoV-2 infection with dyspnea, respiratory rate greater than 30 per min, blood oxygen saturation $\left(\mathrm{SpO}_{2}\right)$ of $93 \%$ or less, $\mathrm{PaO} 2 / \mathrm{FiO}_{2}$ ratio below 300, and/or lung infiltration in more than 50\% of the lung field within 24 to $48 \mathrm{~h}$. Patients with critical disease (respiratory failure requiring mechanical ventilation, septic shock, and/or organ failure requiring intensive care) were not included [17]. The inclusion criteria were: throat swab tested using RT-PCR and confirmed as positive for SARS-CoV-2; complete admission information and clinical data; and moderate or severe COVID-19. The exclusion criteria were: missing or incomplete data; immunologic deficiency or autoimmune disease; excessive use of a corticosteroid or immunosuppressant within 1 month before admission; and pneumonectomy and/or CT motion artifacts. Based on initial chest CT results at admission, the 61 patients were classified into a GGO group $(n=45)$ or a non-GGO group $(\mathrm{n}=16)$. The clinical and laboratory data of these two groups were compared.

\section{Acquisition and analysis of lung CT images}

All included patients received 64-slice CT scans of their lungs on the first day of admission using a SOMATOM Definition AS+ (Siemens Healthineers, Forchheim, Germany). The lung CT scan ranged from the apex to the bottom. Each lung CT result was interpreted by a radiologist with more than 10 years 
experience. The $\mathrm{CT}$ scanning parameters were as follows: tube voltage $=120 \mathrm{kVp}$, pitch $=1.2$, matrix $=$ $512 \times 512$, field of view $=350 \mathrm{~mm} \times 350 \mathrm{~mm}$, and with automatic tube current modulation. All images were then reconstructed with a slice thickness of $1.5 \mathrm{~mm}$ at the same increment [18, 19]. After each patient completed the examination, the testing instrument and the testing room were strictly disinfected with an air sterilizer and $75 \%$ ethanol. The determination GGO of the lungs was in strict accordance with the literature [5].

Table 1. Demographic and clinical characteristics of all 61 COVID-19 patients and of those in the GGO and non-GGO groups.*

\begin{tabular}{lllll}
\hline Characteristic & Total (n=61) & GGO (n=45) & nGGO(n=16) & $P$ value \\
\hline $\begin{array}{l}\text { Demographics } \\
\text { Age (years) }\end{array}$ & & $54.8 \pm 17.8$ & $53 \pm 14.9$ & $0.716^{\mathrm{a}}$ \\
Gender & & & & \\
Female & $32(52.5 \%)$ & $21(46.7 \%)$ & $11(68.7 \%)$ & 0.129 \\
Male & $29(47.5 \%)$ & $24(53.3 \%)$ & $5(31.3 \%)$ & \\
Comorbidities & & & & \\
Fever & $40(65.6 \%)$ & $29(72.5 \%)$ & $11(68.8 \%)$ & $0.756^{\mathrm{b}}$ \\
Any additional disease & $45(73.7 \%)$ & $35(57.3 \%)$ & $10(16.3 \%)$ & $0.102^{\mathrm{b}}$ \\
COPD & $4(6.6 \%)$ & $2(4.4 \%)$ & $2(12.5 \%)$ & 0.267 \\
Cardiovascular disease & $3(4.9 \%)$ & $3(6.7 \%)$ & 0 & 0.294 \\
Cerebrovascular disease & $2(3.3 \%)$ & $2(4.4 \%)$ & 0 & 0.395 \\
Chronic liver disease & $2(3.3 \%)$ & $1(2.2 \%)$ & $1(6.3 \%)$ & 0.441 \\
Diabetes & $11(18.0 \%)$ & $8(17.8 \%)$ & $3(18.8 \%)$ & 0.931 \\
Hypertension & $21(34.4 \%)$ & $16(35.6 \%)$ & $5(31.3 \%)$ & 0.756 \\
Other & $3(4.9 \%)$ & $3(6.7 \%)$ & 0 & 0.294 \\
Severe disease & $5(8.2 \%)$ & $5(11.1 \%)$ & 0 & 0.168 \\
\hline
\end{tabular}

* Categorical data are presented as $\mathrm{n}(\%)$ and continuous data as mean \pm SD. Variables with normal distributions were compared using the Shapiro-Wilk test, and other variables using Fisher's exact test. $a$, independent variables $t$-test; $b$,

Chi-Square test.

\section{Treatments}

All patients were hospitalized and received isolation and treatment in accordance with the therapeutic principles based on the 2019-nCoV guidelines (Trial Version 5) proposed by the National Health Commission of China [20].

\section{Data collection}

Patient demographic data (gender, age, etc.), presence of fever, blood indices (hemoglobin level and counts of white blood cells, neutrophils, lymphocytes, monocytes, and platelets), C-reactive protein (CRP), and immune-related indices (IL-2, -4, -6 , and -10; TNF-a, IFN- $\gamma$; CD3+, CD3+CD4+, and $\mathrm{CD} 3+\mathrm{CD} 8+\mathrm{T}$ cells) were recorded. The presence of comorbidities (hypertension, diabetes, and cerebrovascular diseases) was also recorded.

\section{Statistical analysis}

SPSS version 19.0 software was used for statistical analysis. Continuous variables with normal distributions are presented as means \pm standard deviations (SDs), and those with non-normal distributions as medians and interquartile ranges (IQRs). Count data are presented as numbers and percentages. Comparisons of categorical variables were performed using the chi-square test or using Fisher's exact test if the expected count of at least one cell was less than 5. Comparisons of continuous variables were performed using the $t$-test (if normally distributed) or the Mann-Whitney $U$ test (if not normally distributed). For receiver operating characteristic (ROC) analysis, the optimal cutoff value was the point closest to the upper-left corner of the ROC curve. A multiple logistic regression model was established using categorical data to determine the potential risk factors for GGO by comparing patients with moderate and severe disease. A P value below 0.05 was considered significant.

\section{Results}

\section{Demographic and clinical features of the patients}

We examined 61 patients with moderate or severe COVID-19 during a 2 month period. Based on the CT results, there were 45 patients $(73.8 \%)$ with GGOs group and 16 patients $(26.2 \%)$ without GGOs (Table 1). These two groups had similar mean age (54.8 \pm 17.8 vs. $53 \pm 14.9$ years, $\mathrm{P}=0.716$ ) and similar percentages of males and females $(\mathrm{P}=0.129)$. Hypertension and diabetes were the most common comorbidities, but the two groups did not differ significantly in comorbidities. Among all 61 cases, 5 patients $(8.2 \%)$ had severe disease, all of whom were in the GGO group.

\section{Univariate analyses}

We analyzed 16 different laboratory parameters (Table 2). Univariate analysis showed that age, gender, fever, WBC count, hemoglobin, neutrophil count, lymphocyte count, monocyte count, platelet count, CRP, IL-6, IL-10, TNF-a, CD3+ T cells (\%), CD3+CD8+T cells (\%), and CD3+CD4+T cells (\%) were not significantly different between the GGO and non-GGO groups (all P > 0.05). However, univariate analyses showed that the GGO group had significantly higher levels of IL-2, IL-4, and INF- $\gamma$ (all $\mathrm{P}<0.05)$.

\section{ROC analysis}

We then used each of the 3 indices that were significantly different between the two groups (IL-2, IL-4, IFN- $\gamma$ ) for ROC analysis, with calculations of the area under the curve and optimal cutoff value for each index (Table 3, Fig. 1). The AUC was 0.716 for IL-2, 0.789 for IL-4, and 0.696 for IFN- $\gamma$ and the optimal 
cutoff value was $3.205 \mathrm{pg} / \mathrm{mL}$ for IL-2, $3.175 \mathrm{pg} / \mathrm{mL}$ for IL-4, and $1.875 \mathrm{pg} / \mathrm{mL}$ for IFN- $\gamma$.

\section{Conversion of continuous variables into bi-categorical variables and multivariate logistic regression}

We converted each of the 3 indices (IL-2, IL-4, and IFN- $\gamma$ ) into a bi-categorical variable based on its optimal cutoff value, and then performed multivariable analysis to determine the relationship of each index with GGO (Table 4). The results showed that an IL-2 level above 3.205 was significantly and independently associated with an increased risk of pulmonary GGO (OR: 8.167; 95\% CI: 1.63, 40.8; P < $0.05)$, but there were no significant associations of IL-2 or IFN- $\gamma$ with pulmonary GGO (both $\mathrm{P}>0.05$ ).

Table 2. Univariate analysis of the GGO and non-GGO groups.*

\begin{tabular}{llll}
\hline Variable & GGO $(\mathrm{n}=45)$ & nGGO $(\mathrm{n}=16)$ & $P$ value \\
\hline $\mathrm{WBC}\left(\times 10^{9} / \mathrm{L}\right)$ & $5.37(4.26-6.67)$ & $4.83(3.9-7.49)$ & 0.828 \\
$\mathrm{Hb}(\mathrm{g} / \mathrm{L})$ & $124.6 \pm 13.9$ & $121.5 \pm 14.1$ & $0.453 \mathrm{a}$ \\
$\mathrm{PLT}\left(\times 10^{9} / \mathrm{L}\right)$ & $225.2 \pm 76.7$ & $233.7 \pm 93.3$ & $0.723 \mathrm{a}$ \\
$\mathrm{N}\left(\times 10^{9} / \mathrm{L}\right)$ & $3.26(2.43-4.47)$ & $2.85(1.83-5.19)$ & 0.713 \\
$\mathrm{~L}\left(\times 10^{9} / \mathrm{L}\right)$ & $1.27 \pm 0.5$ & $1.53 \pm 0.67$ & $0.122^{\mathrm{a}}$ \\
$\mathrm{M}\left(\times 10^{9} / \mathrm{L}\right)$ & $0.49(0.38-0.61)$ & $0.57(0.36-0.70)$ & 0.238 \\
$\mathrm{CRP}(\mathrm{mg} / \mathrm{L})$ & $6.91(2.6-29.85)$ & $6.80(3.14-38.2)$ & 0.839 \\
$\mathrm{IL}-2(\mathrm{pg} / \mathrm{mL})$ & $3.3(2.6-4.23)$ & $2.55(2.33-2.94)$ & $\mathbf{0 . 0 1 2}$ \\
$\mathrm{IL}-4(\mathrm{pg} / \mathrm{mL})$ & $2.93(2.11-4.47)$ & $2.24(1.63-2.89)$ & $\mathbf{0 . 0 2 9}$ \\
$\mathrm{IL}-6(\mathrm{pg} / \mathrm{mL})$ & $8.83(5.02-20.34)$ & $5.70(3.69-26.86)$ & 0.258 \\
$\mathrm{IL}-10(\mathrm{pg} / \mathrm{mL})$ & $4.58(3.86-5.54)$ & $4.27(2.91-4.64)$ & 0.081 \\
$\mathrm{TNF}-\mathrm{a}(\mathrm{pg} / \mathrm{mL})$ & $3.11(2.13-4.38)$ & $2.37(1.93-3.67)$ & 0.194 \\
IFN- $(\mathrm{pg} / \mathrm{mL})$ & $3.02(2.25-3.88)$ & $2.06(1.75-2.47)$ & $\mathbf{0 . 0 2 3}$ \\
$\mathrm{CD} 3+\mathrm{CD} 8+\mathrm{T}(\%)$ & $25.1 \pm 9.2$ & $36.3 \pm 49.7$ & $0.183 \mathrm{a}$ \\
$\mathrm{CD} 3+\mathrm{T}(\%)$ & $76.44(72.34-80.2)$ & $79.9(70.4-81.87)$ & 0.726 \\
$\mathrm{CD} 3+\mathrm{CD} 4+\mathrm{T}(\%)$ & $46.54(41.28-51.5)$ & $48.2(41.06-54.28)$ & 0.705
\end{tabular}

*Data are given as mean \pm SD or median (IQR). WBC, white blood cells; $\mathrm{Hb}$, hemoglobin; PLT, platelets; N, neutrophils; L, lymphocytes; M, monocytes.

Gender was analyzed using the Pearson Chi-square test; Hb, PLT, N, L, and CD3+CD8+ T cells using the Welch $t$ test; and other variables using the

Mann-Whitney U-rank sum test. a, independent variable $t$-test; all others,

Mann-Whitney U test.

Table 3. AUCs and optimal cutoff values of different indices from ROC analyses of IL-2, IL-4, and IFN-y for the determination of GGO (see Figure 1).

\begin{tabular}{lllllll}
\hline Variable & AUC & 95\%CI & \multicolumn{3}{c}{ Sensitivity Specificity $\begin{array}{l}\text { Optimal cutoff } \\
(\mathrm{pg} / \mathrm{mL})\end{array}$} & $\begin{array}{l}P \\
\text { value }\end{array}$ \\
\hline IL-2 & 0.716 & $0.569-0.864$ & 0.538 & 0.875 & 3.205 & $\mathbf{0 . 0 1 2}$ \\
IL-4 & 0.689 & $0.549-0.829$ & 0.487 & 0.875 & 3.175 & $\mathbf{0 . 0 2 9}$ \\
IFN-ץ & 0.696 & $0.525-0.868$ & 0.897 & 0.375 & 1.875 & $\mathbf{0 . 0 2 3}$ \\
\hline
\end{tabular}

AUC: area under the curve; $\mathrm{CI}$ : confidence interval.

Table 4. Multivariate logistic regression analysis of 3 bi-categorical variables for the determination of GGO.

\begin{tabular}{llll}
\hline Variable & Cutoff value & OR $(95 \% \mathrm{CI})$ & P-value \\
\hline IL-2 $(\mathrm{pg} / \mathrm{mL})$ & $>3.205$ & $8.167(1.633-40.848)$ & 0.011 \\
$\mathrm{IL}-4(\mathrm{pg} / \mathrm{mL})$ & $>1.875$ & $2.625(1.106-32.537)$ & 0.218 \\
IFN-ץ $(\mathrm{pg} / \mathrm{mL})$ & $>3.175$ & $3.864(0.489-30.531)$ & 0.200 \\
\hline
\end{tabular}

OR: odds ratio; $\mathrm{CI}$ : confidence interval

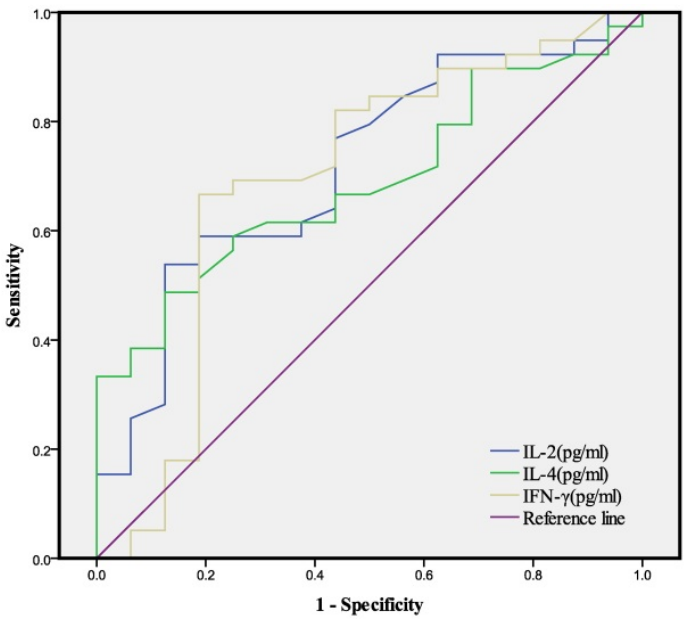

Figure 1. ROC analysis of IL-2, IL-4, and IFN-y for the determination of GGO (see Table 3).

\section{Discussion}

The present retrospective case-control study of 61 patients with moderate or severe COVID-19 compared 45 patients who had GGOs in the lungs with 16 patients who had no GGOs. Our analysis of clinical and other data collected at admission indicated that the levels of three cytokines - IL-2, IL-4, and IFN- $\gamma$ - had positive correlations with the presence of GGOs. Our subsequent multivariate logistic regression analysis showed that IL-2 was a significant and independent risk factor for GGOs patients with moderate or severe COVID-19.

GGO in a CT image is defined by the presence of increased pulmonary opacity and preservation of the bronchial and vascular margins, different from the increased homogeneity of pulmonary consolidations and burr signs in blood vessels and airway walls [11]. GGO is a common and nonspecific finding in chest $X$-rays and $\mathrm{CT}$, and occurs in patients with many pulmonary diseases [21]. Pneumonia and other diseases are characterized by lung damage in which the inflammatory cells suffuse alveolar spaces and walls, and pulmonary interstitial thickening leads to an increased density relative to air, manifesting as GGO in a CT image [22]. The radiographic imaging results of patients with SARS-CoV-2 are variable, and there may be pulmonary consolidation, subsegmental pulmonary vasodilation, interlobular septa thickening, with or without air bronchogram, but GGO is the most common finding from pulmonary CT [23]. Although GGO cannot be considered pneumonia-specific for COVID-19 in CT qualitative analysis, quantitative analysis of GGOs and fibrosis alterations on chest CT can identify COVID-19 patients [24].

Previous research [25] showed that the CT abnormalities in patients with COVID-19 pneumonia 
were more obvious than in those with non-COVID-19 pneumonia, in that the COVID-19 group had a higher frequency of GGO (91\% vs. $68 \%, \mathrm{P}<0.001)$. Another study [26] compared patients hospitalized with acute respiratory distress syndrome (ARDS) caused by COVID-19 or H1N1 and also found that the lung CTs of the COVID-19 patients had a higher frequency of GGO (94.5\% vs. $45.3 \%)$. SARS-CoV-2 infection induces an excessive and prolonged cytokine/chemokine response in some individuals - a cytokine storm. An uncontrolled cytokine storm can lead to ARDS or multiple organ dysfunction (MOD), and ultimately to physiological deterioration and death [27]. Other studies of COVID-19 patients reported that their CT images had a characteristic GGO, indicating that the lungs contained fluid [28]. A recent case report, which described the autopsy of a patient with COVID-19, confirmed that their lungs were filled with a transparent liquid jelly-like substance, similar to that in the lungs of drowning victims [12]. Although the nature of this substance has yet to be determined, a study of COVID-19 patients showed it was due to the cytokine storm that promoted pneumonia and pulmonary GGO [29]. At present, the relationship of the cytokine storm with the progression of COVID-19 and how it causes GGO of the lungs is still unclear. This remains an important topic that requires additional research.

We examined 61 patients with moderate-tosevere COVID-19 in this study. CT of their lungs showed that $73.8 \%$ of them had GGO, consistent with the findings of previous studies. We found that the lung CTs of patients with moderate or severe pneumonia due to COVID-19 were more likely to have GGO if they had increased levels of IL-2, IL-4, and INF- $\gamma$, and that IL-2 was significantly and independently associated with GGO. These results are consistent with previous research which reported that the cytokine storm in patients with COVID-19 pneumonia was associated with increased levels of IL-2 and INF- $\gamma$ [30]. Other researchers also reported elevated levels of IL-2, IL-4, and INF- $\gamma$ in patients with COVID-19 [31, 32], and that these levels were highly correlated with disease progression. In addition, critically ill COVID-19 patients had significantly higher levels of IL-2, IL-4, and INF- $\gamma$ than those with moderate or severe disease [33, 34], and those with severe COVID-19 had higher levels of certain cytokines than those with mild disease [32]. This confirms that the severity of the cytokine storm is related to disease severity [32]. In support of this interpretation, a previous study of COVID-19 patients showed that intravenous injection of mesenchymal stem cells (MSCs) reduced the pulmonary GGO and the infiltration of inflammatory cells in the lungs by inhibiting the cytokine storm and by promoting tissue repair and regeneration [16]. Based on our results and these previous studies, we speculate that IL-2, IL-4, and INF- $\gamma$ are involved in the infiltration of alveolar inflammatory cells and lung injury caused by COVID-19. Although our GGO group had greater levels of IL-2, IL-4, and INF- $\gamma$ than the non-GGO group, the mechanism by which these inflammatory factors cause ARDS and lung injury is still unclear.

A total of $86.9 \%$ of our patients had significantly elevated IL-6 levels, consistent with previous reports [35]. An elevated serum IL-6 level is common in patients with severe COVID-19 [34]. Chen et al. [36] reported that the serum SARS-CoV-2 viral load in critically ill patients was highly correlated with the serum IL-6 level $(R=0.902)$. High levels of IL-6 may also cause an increase in neutrophils and a decrease in lymphocytes [37]. Moreover, IL-6 may promote the development of ARDS in COVID-19 patients, and an increased IL-6 level may be a sign of more aggravated disease [37]. In contrast, we found no statistical difference in the IL-6 levels in our GGO and non-GGO groups. The reason for this discrepancy may that we only examined patients with moderate or severe disease, and excluded critically ill patients. Thus, we are planning follow-up clinical studies that have larger sample sizes to compare the data of patients with different disease severities and clarify the mechanism of the formation of lung GGOs in patients with COVID-19.

This study has certain limitations. In particular, this was a retrospective single-center study and the number of cases was relatively small. A large multi-center study would provide more reliable results. In addition, we did not examine patients with other viral infections. Analysis of the relationship between lung CT findings and cytokine levels of patients with other viral infections may help us distinguish COVID-19 pneumonia from other lung infections. We also did not study the relationship between CT results and the levels of cytokines during the initial stage of infection nor in patients with asymptomatic SARS-CoV-2 infections. We believe that further studies of the relationship between changes in CT images and the levels of cytokines may help to monitor and predict the course of disease, and may also provide support for clinical decision-making.

\section{Conclusion}

We propose that the appearance of GGOs in the CT images of patients with moderate-to-severe COVID-19 is related to increased levels of certain cytokines, and that IL-4 and INF- $\gamma$ play key roles in causing the cytokine storm and GGOs in these 
patients. Moreover, we found that IL-2 was an independent risk factor for lung GGOs in patients with moderate-to-severe COVID-19. This suggests a possible new approach for studying the mechanism of lung disease in COVID-19.

\section{Abbreviations}

COVID-19: coronavirus disease 2019; GGO: Ground-glass opacity; SARS: severe acute respiratory syndrome; MERS: Middle East respiratory syndrome; CT: computed tomography; ARDS: acute respiratory distress syndrome; WBC: white blood cell; $\mathrm{Hb}$ : hemoglobin; PLT: platelet; $\mathrm{N}$ : neutrophil; $\mathrm{L}$ : lymphocyte; M: monocyte; CRP: C-reactive protein; IL-2: Interleukin-2; IL-4: Interleukin-4; IL-6: Interleukin-6; IL-10: Interleukin-10; TNF-a: Tumor necrosis factor- $\alpha$; INF- $\gamma$ : Interferon- $\gamma$; ROC: receiver operating characteristic; AUC: area under the curve.

\section{Acknowledgements}

This research was funded by Health Commission of Hubei Province scientific research project (No.WJ2021M239), supported by the Hubei Natural Science Foundation of China (No.2020CFB764) and supported by the National Natural Science Foundation of China (No.81500218, No.81601324). We thank Professor Jing Wu (Department of Epidemiological Statistics, Tongji Medical College, Huazhong University of Science and Technology) for providing statistical guidance. We also thank the frontline medical staff for their dedication.

\section{Author Contributions}

Zubo $\mathrm{Wu}$, Xiaoping Liu, Jie Liu designed the study, performed the data analysis and drafted the initial manuscript. Feng $\mathrm{Zhu}$ designed the data collection instruments, collected the data. Hua Peng, Yalan Liu, Yali Liu supervised the data collection, reviewed and revised the manuscript. All authors read and approved the final manuscript.

\section{Competing Interests}

The authors have declared that no competing interest exists.

\section{References}

1. Zhu N, Zhang D, Wang W, Li X, Yang B, Song J, et al. A Novel Coronavirus from Patients with Pneumonia in China, 2019. N Engl J Med. 2020; 382: 727-33.

2. Chen N, Zhou M, Dong X, Qu J, Gong F, Han Y, et al. Epidemiological and clinical characteristics of 99 cases of 2019 novel coronavirus pneumonia in Wuhan, China: a descriptive study. Lancet. 2020; 395: 507-13.

3. Baden LR, Rubin EJ. Covid-19 - The Search for Effective Therapy. N Engl J Med. 2020; 382: 1851-2.

4. Watanabe M, Caruso D, Tuccinardi D, Risi R, Zerunian M, Polici M, et al. Visceral fat shows the strongest association with the need of intensive care in patients with COVID-19. Metabolism. 2020; 111: 154319.

5. Chung M, Bernheim A, Mei X, Zhang N, Huang M, Zeng X, et al. CT Imaging Features of 2019 Novel Coronavirus (2019-nCoV). Radiology. 2020; 295: 202-7.
6. Wu J, Wu X, Zeng W, Guo D, Fang Z, Chen L, et al. Chest CT Findings in Patients With Coronavirus Disease 2019 and Its Relationship With Clinical Features. Invest Radiol. 2020; 55: 257-61.

7. Li K, Wu J, Wu F, Guo D, Chen L, Fang Z, et al. The Clinical and Chest CT Features Associated With Severe and Critical COVID-19 Pneumonia. Invest Radiol. 2020; 55: 327-31.

8. Guan WJ, Ni ZY, Hu Y, Liang WH, Ou CQ, He JX, et al. Clinical Characteristics of Coronavirus Disease 2019 in China. N Engl J Med. 2020; 382: 1708-20.

9. Pan Y, Guan H, Zhou S, Wang Y, Li Q, Zhu T, et al. Initial CT findings and temporal changes in patients with the novel coronavirus pneumonia (2019-nCoV): a study of 63 patients in Wuhan, China. Eur Radiol. 2020; 30: 3306-9.

10. Duan YN, Qin J. Pre- and Posttreatment Chest CT Findings: 2019 Novel Coronavirus (2019-nCoV) Pneumonia. Radiology. 2020; 295: 21.

11. Hansell DM, Bankier AA, MacMahon H, McLoud TC, Muller NL, Remy J. Fleischner Society: glossary of terms for thoracic imaging. Radiology. 2008; 246: 697-722.

12. Xu Z, Shi L, Wang Y, Zhang J, Huang L, Zhang C, et al. Pathological findings of COVID-19 associated with acute respiratory distress syndrome. Lancet Respir Med. 2020; 8: 420-2.

13. Sun X, Wang T, Cai D, Hu Z, Chen J, Liao H, et al. Cytokine storm intervention in the early stages of COVID-19 pneumonia. Cytokine Growth Factor Rev. 2020; 53: 38-42.

14. Huang $\mathrm{C}$, Wang $\mathrm{Y}$, $\mathrm{Li} \mathrm{X}$, Ren $\mathrm{L}$, Zhao J, Hu Y, et al. Clinical features of patients infected with 2019 novel coronavirus in Wuhan, China. Lancet. 2020; 395: 497-506.

15. Yuan Y, Wang QP, Sun D, Wu ZB, Peng H, Liu XW, et al. Differences in Immune Responses between Children and Adults with COVID-19. Curr Med Sci. 2021; 41: 58-61.

16. Leng Z, Zhu R, Hou W, Feng Y, Yang Y, Han Q, et al. Transplantation of ACE2(-) Mesenchymal Stem Cells Improves the Outcome of Patients with COVID-19 Pneumonia. Aging Dis. 2020; 11: 216-28.

17. [Internet]. WHO. Report of the WHO -China Joint Mission on Coronavirus Disease 2019 (COVID -19). WHO; 2020. https://www.who.int/docs/defaultsource/coronaviruse/who-china-joint-mission-on-covid-19-final-report.pdf.

18. Shi H, Han X, Jiang N, Cao Y, Alwalid O, Gu J, et al. Radiological findings from 81 patients with COVID-19 pneumonia in Wuhan, China: a descriptive study. Lancet Infect Dis. 2020; 20: 425-34.

19. Li L, Yang L, Gui S, Pan F, Ye T, Liang B, et al. Association of clinical and radiographic findings with the outcomes of 93 patients with COVID-19 in Wuhan, China. Theranostics. 2020; 10: 6113-21.

20. [Internet]. National Health Commission of the People's Republic of China. Notice on the issuance of a new coronavirus pneumonia diagnosis and treatment plan (try the fifth revised version). National Health Commission of the People's Republic of China; 2020. http://www.nhc.gov.cn/yzygi/s7653p/ 202002/d4b895337e19445f8d728fcaf1e3e13a.shtml.

21. El-Sherief AH, Gilman MD, Healey TT, Tambouret RH, Shepard JA, Abbott $\mathrm{GF}$, et al. Clear vision through the haze: a practical approach to ground-glass opacity. Curr Probl Diagn Radiol. 2014; 43: 140-58.

22. Collins J, Stern EJ. Ground-glass opacity at CT: the ABCs. AJR Am J Roentgenol. 1997; 169: 355-67.

23. Caruso D, Polidori T, Guido G, Nicolai M, Bracci B, Cremona A, et al. Typical and atypical COVID-19 computed tomography findings. World J Clin Cases. 2020; 8: 3177-87.

24. Caruso D, Polici M, Zerunian M, Pucciarelli F, Polidori T, Guido G, et al. Quantitative Chest CT analysis in discriminating COVID-19 from non-COVID-19 patients. Radiol Med. 2021; 126: 243-9.

25. Bai HX, Hsieh B, Xiong Z, Halsey K, Choi JW, Tran TML, et al. Performance of Radiologists in Differentiating COVID-19 from Non-COVID-19 Viral Pneumonia at Chest CT. Radiology. 2020; 296: E46-E54.

26. Tang X, Du RH, Wang R, Cao TZ, Guan LL, Yang CQ, et al. Comparison of Hospitalized Patients With ARDS Caused by COVID-19 and H1N1. Chest. 2020; 158: 195-205.

27. Ye Q, Wang B, Mao J. The pathogenesis and treatment of the 'Cytokine Storm' in COVID-19. J Infect. 2020; 80: 607-13

28. Wang D, Hu B, Hu C, Zhu F, Liu X, Zhang J, et al. Clinical Characteristics of 138 Hospitalized Patients With 2019 Novel Coronavirus-Infected Pneumonia in Wuhan, China. Jama. 2020; 323: 1061-9.

29. Shi Y, Wang Y, Shao C, Huang J, Gan J, Huang X, et al. COVID-19 infection: the perspectives on immune responses. Cell Death Differ. 2020; 27: 1451-4.

30. Mallis P, Michalopoulos E, Chatzistamatiou T, Stavropoulos-Giokas C. Mesenchymal stromal cells as potential immunomodulatory players in severe acute respiratory distress syndrome induced by SARS-CoV-2 infection. World J Stem Cells. 2020; 12: 731-51.

31. Guan J, Wei X, Qin S, Liu X, Jiang Y, Chen Y, et al. Continuous tracking of COVID-19 patients' immune status. Int Immunopharmacol 2020; 89: 107034

32. Liu J, Li S, Liang B, Wang X, Wang H, Li W, et al. Longitudinal characteristics of lymphocyte responses and cytokine profiles in the peripheral blood of SARS-CoV-2 infected patients. EBioMedicine. 2020; 55: 102763.

33. Zhao Y, Zhou J, Pan L, Zhang Y, Wang H, Wu W, et al. Detection and analysis of clinical features of patients with different types of coronavirus disease 2019. J Med Virol. 2020.

34. Zhou F, Yu T, Du R, Fan G, Liu Y, Liu Z, et al. Clinical course and risk factors for mortality of adult inpatients with COVID-19 in Wuhan, China: a retrospective cohort study. Lancet. 2020; 395: 1054-62. 
35. Paces J, Strizova Z, Smrz D, Cerny J. COVID-19 and the immune system. Physiol Res. 2020; 69: 379-88.

36. Chen X, Zhao B, Qu Y, Chen Y, Xiong J, Feng Y, et al. Detectable Serum Severe Acute Respiratory Syndrome Coronavirus 2 Viral Load (RNAemia) Is Closely Correlated With Drastically Elevated Interleukin 6 Level in Critically Ill Patients With Coronavirus Disease 2019. Clin Infect Dis. 2020; 71: 1937-42.

37. Wang J, Jiang M, Chen X, Montaner LJ. Cytokine storm and leukocyte changes in mild versus severe SARS-CoV-2 infection: Review of 3939 COVID-19 patients in China and emerging pathogenesis and therapy concepts. J Leukoc Biol. 2020; 108: 17-41. 